Article

\title{
The Contribution of Geomagnetic Activity to Ionospheric foF2 Trends at Different Phases of the Solar Cycle by SWM
}

\author{
Haimeng $\mathrm{Li}^{1}{ }^{1}$, Jing-Song Wang ${ }^{2}$, Zhou Chen ${ }^{1,2, *}$, Lianqi Xie ${ }^{1}$, Fan $\mathrm{Li}^{1}$ and Tongji Zheng ${ }^{1}$ \\ 1 Institute of Space Science and Technology, Nanchang University, Nanchang 330031, China; \\ lihaimeng@ncu.edu.cn (H.L.); Xielianqi1@163.com (L.X.); LIFAN9863@163.com (F.L.); \\ zhengtongji@163.com (T.Z.) \\ 2 Key Laboratory of Space Weather, National Center for Space Weather, China Meteorological Administration, \\ Beijing 100081, China; wangjs@cma.gov.cn \\ * Correspondence: chenzhou760@foxmail.com
}

Received: 26 April 2020; Accepted: 8 June 2020; Published: 11 June 2020

check for updates

\begin{abstract}
Solar activity dominates the temporal variability of ionospheric properties, which makes it difficult to identify and isolate the effects of geomagnetic activity on the ionosphere. Therefore, the latter effects on the ionosphere are still unclear. Here, we use the spectral whitening method (SWM) - a proven approach to extract ionospheric perturbations caused by geomagnetic activity-to directly obtain, in isolation, the effects of geomagnetic activity. We study its contribution to the ionosphere for different phases of the solar cycle. The time lag between the solar and geomagnetic activities provides an opportunity to understand the contribution of geomagnetic activity to the perturbation of the ionosphere. The results suggest that this contribution to the ionosphere is significant when geomagnetic activity is at its maximum level, which usually happens in the declining phase of the solar cycle, but the contribution is very weak at the solar minimum and during the ascending phase. Then, by analyzing the contributions in different months, we find that the role of geomagnetic activity is larger around winter but smaller around summer.
\end{abstract}

Keywords: ionospheric properties; spectral whitening method; geomagnetic activity; phase of solar cycle

\section{Introduction}

Ionospheric time variation has been studied for many years. It is driven by many mechanisms, such as neutral wind, solar, and geomagnetic activities, and particle precipitation driven by wave-particle interactions [1-7]. Many researchers have studied the effects of solar activity on the ionospheric trend variation [6,7]. Some studies have used particular observations to analyze the ionospheric trend variations, including the upper ionospheric electron density [8], the ionospheric temperature [9], and even differential GPS code biases [10]. In addition, a range of observations have been used to study ionospheric cooling, which can be summarized in several ways, including by analyzing the decrease in the peak height of the ionospheric F2 layer and the upper ionospheric density [11]. It is well known that solar activity strongly affects the ionosphere and dominates ionospheric variability with local time (LT) [6], so its effect on ionospheric trend variation is highly significant. These well-known solar-activity-induced variations make it difficult to detect and extract trend variations caused by other drivers, among which geomagnetic activity is also a very important driver [12]. As reported by Mikhailov and Marin [13], studies on trend variations in the critical frequency of the ionospheric F2 layer $(f o F 2)$ should not neglect the effects of geomagnetic activity. 
Thus, it is important to develop an effective approach to characterize the effects of geomagnetic activity on the ionosphere. In fact, application of different methods usually leads to interesting but puzzling results $[14,15]$. Therefore, finding a suitable approach is a focus in this field of research. Some authors have suggested that the effects of geomagnetic activity on the ionospheric trend cannot be extracted effectively using traditional methods, such as based on conventional monthly or annual mean $A p$-index measurements, or analysis of geomagnetic activity indices [13].

This implies that different authors use different approaches to extract trend variations from the ionospheric observations and that the conclusions of analysis greatly depend on the adopted methods [15]. Researchers have used various novel mathematic methods to explore new geophysical mechanisms, such as the numerical differentiation method [16] and spectral analysis method [17]. In general, the useful extracted "signal" (such as the effect of geomagnetic activity) is very small and the "background" is very noisy, so special methods are required to reveal the perturbation driven by the "signal" in the observed foF2 trend variations. The spectral whitening method (SWM), first introduced by Wang et al. [18], may be an accurate and effective method for characterizing the effects of geomagnetic activity on ionospheric $f o \mathrm{~F} 2$ observations. As a mathematical method, it does not depend on input parameters such as geomagnetic indices (e.g., Ap). Ionospheric perturbations derived from the SWM are not affected by residual effects owing to solar activity during geomagnetically quiet periods. This approach can, therefore, remove the effects of solar activity more effectively than the conventional method.

In this paper, the SWM is used to study the contributions of geomagnetic activity to the ionospheric trends. The plausible causes of the contributions will be discussed at various phases of the solar cycle. Our research shows that the effects of geomagnetic activity on the ionosphere greatly vary during different phases of the solar cycle. The $f o \mathrm{~F} 2$ ionospheric is most notably affected by geomagnetic activity during the declining phase of the solar activity, especially around winter.

\section{Data and Methods}

\subsection{Data}

Our analysis covers high to low latitudes in both hemispheres. Twenty-seven ionosonde stations (see Table 1) located between geographical latitudes $+67.4^{\circ} \mathrm{N}$ and $-67.6^{\circ} \mathrm{S}$ were selected because (1) they were as widely distributed as possible and (2) the $f \circ \mathrm{F} 2$ observations cover at least 30 years. The common period covered by the data for all stations ranges from 1959 to 1990, with a time resolution of $1 \mathrm{~h}$.

F10.7 is the solar radio flux at a wavelength of $10.7 \mathrm{~cm}$ measured for the earth's orbit, which is most widely used as an index of solar activity. This index is generated daily at the National Center for Environmental Information (NOAA). The Ap index is obtained from the geomagnetic field measurements at a number of stations. It is usually used as a criterion of the general level of geomagnetic activity over the globe for a given day. In the research, in order to analyze the relationship between the foF2 and F10.7/Ap indices, both the original F10.7 and Ap index data are processed by nearest neighbor interpolation to obtain the data with 1-h time resolution.

The aa index, expressed in units of $1 \mathrm{nT}$, is a global geomagnetic activity index with a time resolution of 1 day. The advantage of the aa index is that its time series reaches further back to 1868 and covers a longer period than any of the other geomagnetic activity index time series. It is derived from the $\mathrm{K}$ indices obtained from two approximately antipodal observatories. Because it is based on data obtained from only two observatories, it is the simplest of all the 3 hourly planetary indices. The index strongly correlates with the $a p$ and $a m$ indices. In addition, in this study the solar activity level is represented by the monthly averages of the daily sunspot number. 
Table 1. List of ionosonde stations.

\begin{tabular}{ccccc}
\hline Number & Station & Period & Latitude $\left({ }^{\circ} \mathbf{N}\right)$ & Longitude $\left({ }^{\circ} \mathbf{E}\right)$ \\
\hline 0 & Mawson & $1958-2000$ & -67.6 & 62.9 \\
1 & Port Stanley & $1957-1990$ & -51.7 & -57.8 \\
2 & Hobart & $1950-2007$ & -42.9 & 147.3 \\
3 & Canberra & $1950-2007$ & -35.3 & 149.0 \\
4 & Mundaring & $1959-2007$ & -32.0 & 116.2 \\
5 & Townsville & $1951-2007$ & -19.6 & 146.9 \\
6 & Huancayo & $1957-1990$ & -12.1 & -75.2 \\
7 & Maui & $1957-1990$ & +20.8 & -156.3 \\
8 & Chungli & $1959-1990$ & +24.9 & 121.4 \\
9 & Yamagawa & $1957-1990$ & +31.2 & 130.6 \\
10 & Kokubunji & $1957-1990$ & +35.7 & 139.5 \\
11 & Akita & $1957-1990$ & +39.7 & 140.1 \\
12 & Boulder & $1958-1990$ & +40.0 & -105.3 \\
13 & Alma Ata & $1957-1990$ & +43.2 & 76.9 \\
14 & Wakkanai & $1957-1990$ & +45.4 & 141.7 \\
15 & Dourbes & $1957-1990$ & +50.1 & 4.6 \\
16 & Irkutsk & $1957-1992$ & +52.5 & 104.0 \\
17 & Juliusruh-Ruegen & $1957-2012$ & +54.6 & 13.4 \\
18 & Moscow & $1957-1995$ & +55.5 & 37.3 \\
19 & Gorky & $1958-1990$ & +56.1 & 44.3 \\
20 & Tomsk & $1957-1994$ & +56.5 & 84.9 \\
21 & Sverdlovsk & $1957-1995$ & +56.4 & 58.6 \\
22 & Churchill & $1957-1996$ & +58.8 & -94.2 \\
23 & Uppsala & $1957-1990$ & +59.8 & 17.6 \\
24 & Yakutsk & $1957-1991$ & +62.0 & 129.6 \\
25 & Lycksele & $1957-1990$ & +64.7 & 18.8 \\
26 & Sodankyla & $1957-1990$ & +67.4 & 26.6 \\
\hline & & & &
\end{tabular}

\subsection{Spectral Whitening Method}

The SWM is one kind of signal processing technique used for statistical estimation and detection, which is adopted to detect and remove the periodic disturbances from the background signal or data. Additionally, the detected disturbances by SWM are characterized by a Gaussian-type probability density distribution over all timescales. This process further simplifies statistical analysis and means that the disturbances derived by SWM may have occurred regardless of the underlying physical processes. As a result, the SWM is suitable for both decorrelating a data sequence or controlling the spectral shape. Comparing to some traditional methods, such as the monthly median method (MMM), the SWM has been proven to much more successfully identify and extract the aperiodic disturbance in the background ionosphere. Wang et al. [18] suggested that the SWM-derived disturbance in the ionosphere is more sensitive to external geophysical perturbations and geomagnetic storms. Chen et al. [19] proved that the SWM is very effective for extracting the effects of geomagnetic activity from ionospheric observations. In this study, the SWM is adopted to remove the periodicities in foF2 variations by flattening the Fourier spectrum, and therefore to identify the aperiodic disturbances driven by the geomagnetic activity. The SWM is a filtering method that is used to identify ionospheric perturbations caused by geomagnetic activity. For a time series, $g(t)$, the SWM algorithm reads:

$$
\left\{\begin{array}{l}
g_{d}^{*}(t)=\frac{1}{2 \pi} \int_{-\infty}^{+\infty}\left[\int_{-\infty}^{+\infty} g(t) \times e^{-2 \pi i t \xi} d t\right] \times \frac{P_{0}}{P_{e n v}(\xi)} \times e^{2 \pi i t \xi} d \xi \\
g_{s}\left(t_{m}\right)=\frac{1}{3} \sum_{j=0}^{2} g_{d}^{*}\left(t_{m+j-1}\right)
\end{array}\right.
$$

where $P_{\text {env }}(\xi)$ is the upper envelope of the power spectrum of $g(t)$. The $P_{\text {env }}(\xi)$ can be calculated using the complicated envelope function [20], which can give a good approximation by using the 
maximum value in a given data window. $P_{0}$ is the value that appears most often in the dataset of $P_{e n v}(\xi)$, i.e., the mode of $P_{e n v}(\xi)$. The transformation above actually consists of four steps: (i) A Fourier transform, shown as the bracketed part in Equation (1), is applied to the original data. (ii) The Fourier power spectrum is divided by its upper envelope $P_{e n v}(\xi)$ and the maximum intensity is now unified, so the spectrum is flattened and displays the spectral form of white noise. Actually, the aperiodic components are not discernible in the spectrum; thus, they are merely affected by the whitening process. (iii) The whitened spectrum is multiplied by $P_{0}$ to ensure the most common values of the spectrum intensity before and after the whitening are the same. (iv) A new time series, $g_{d}^{*}(t)$, is derived by using an inverse Fourier transform in the whitened spectrum from step (iii). However, the whitening process causes extra random dithers. Therefore, in order to reduce the enhanced noise, a three-point running average is applied to smooth $g_{d}^{*}(t)$ and the final result of SWM is $g_{\mathrm{s}}\left(t_{\mathrm{m}}\right)$.

In this study, for a given ionospheric station, $g(t)$ denotes the time variation of $f \circ \mathrm{F} 2$. The SWM has been shown to be more effective than the monthly median method (MMM) in the identification of ionospheric perturbations related to geomagnetic activity [19,21,22]. As shown in Figure 1, a strong geomagnetic storm occurred in March 1982. The ionospheric index Dr is defined as the mean of the absolute perturbation values of the global RfoF2 (relative value of the $f \circ \mathrm{F} 2$ ) based on the SWM or MMM at the same time; it represents the overall level of global ionospheric perturbations. It suggests that the $\mathrm{Dr}(\mathrm{SWM})$ index is very effective in responding to global ionospheric perturbations before and after a geomagnetic storm. Regards the Dr (MMM) index, its response to geomagnetic storms can also be observed (shaded periods A and B in Figure 1), but many more strong and significant perturbations appear in shaded period $C$. This disturbance shows significant diurnal variation, which can be observed during the period ranging from 27 February to 9 March. Those disturbances should be the residuals of the diurnal variation, which is caused by the drawback of MMM. The dotted lines in Figure 1 represent the smoothed Dr. Since MMM is one kind of low-pass filtering method and as it is hard to filter the diurnal variation and 27-day quasi-periodicity, the $\operatorname{Dr}(\mathrm{MMM})$ values significantly fluctuate around the smoothed Dr (MMM) over a 27-day cycle.

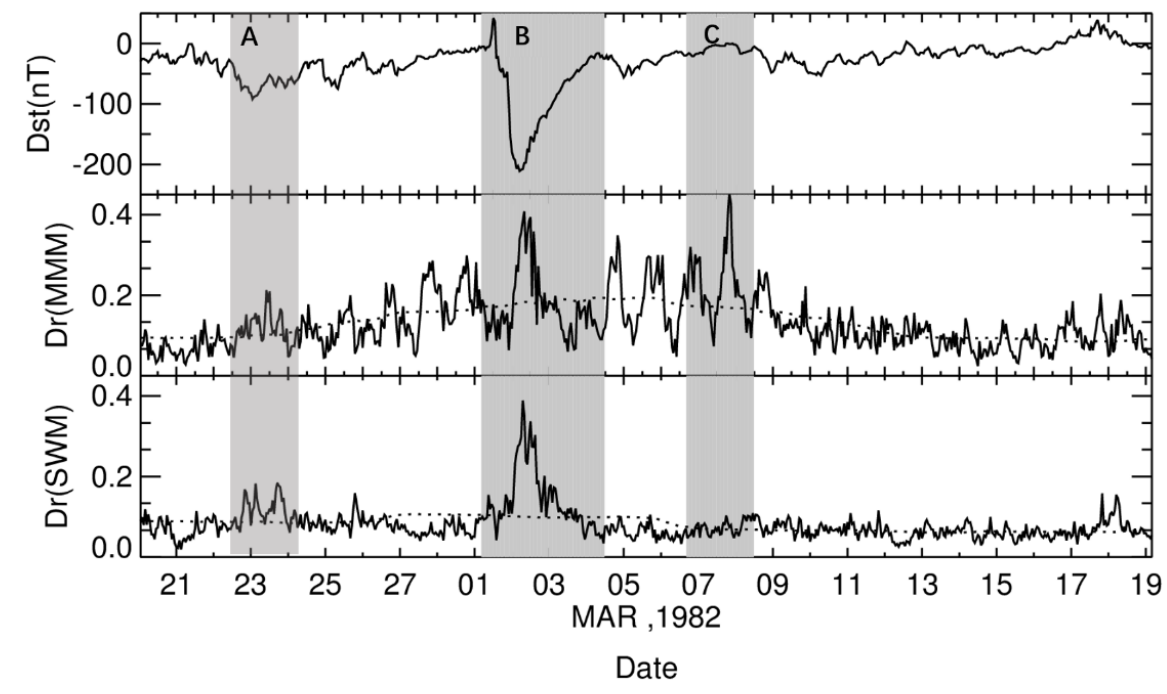

Figure 1. From top to bottom: Dst geomagnetic index, ionospheric index $\operatorname{Dr}(\mathrm{MMM})$, and ionospheric index $D r(\mathrm{SWM})$ indices. The dotted lines represent smoothed $D r$ trends. The scale values in the $x$-axis mean the 00:00 UT (universal time) in one day. The shade of (A) and (B) represent the period of geomagnetic disturbance; the shade of $(C)$ denotes geomagnetic quiet time.

\subsection{Traditional Approach to Isolating Solar Activity}

To study ionospheric trends without interference from the effects of solar activity, a traditional method was adopted. Firstly, a linear regression between the original $f_{o} \mathrm{~F} 2$ and the F10.7 was calculated. This step estimated the effect of solar activity on the ionosphere. Then, the $f_{0} \mathrm{~F} 2$ residuals were 
obtained between the original foF2 and the regression, which meant removing the effect of solar activity from the ionospheric observation. Many researchers have attempted to use different solar activity indices, such as $\mathrm{Rz}$ (solar sunspot number) and the daily F10.7, as proxies for extreme ultraviolet radiation (EUV) $[12,23,24]$. In general, estimation using F10.7 yields better results than that from $\mathrm{Rz}$ [25]. Therefore, in this study the effect of solar activity was isolated by estimating the foF2 residuals from a linear regression between $f \circ \mathrm{F} 2$ and the solar activity measured by the F10.7 index:

$$
\begin{gathered}
\Delta f o \mathrm{~F} 2=f o \mathrm{~F} 2-(a \times \mathrm{F} 10.7+b) \\
\Delta f o \mathrm{~F} 2=\alpha \times \text { year }+\beta
\end{gathered}
$$

where $\mathrm{a}$ and $\mathrm{b}$ are obtained from the regression between foF2 and F10.7 using least-squares minimization. The linear foF2 trend $\alpha$ was then obtained from Equation (3).

The SWM can directly isolate the effects of geomagnetic activity from ionospheric observations:

$$
\begin{gathered}
\Delta f o \mathrm{~F}^{\prime}=\operatorname{SWM}(f o \mathrm{~F} 2) \\
\Delta f o \mathrm{~F}^{\prime}=\alpha^{\prime} \times \text { year }+\beta^{\prime}
\end{gathered}
$$

where SWM (foF2) refers to SWM use in extracting the effects of geomagnetic activity from $f_{0} \mathrm{~F} 2$ following Equation (1). Here, $\alpha$ and $\alpha^{\prime}$ are expressed in units of $\mathrm{MHz}$ year ${ }^{-1} ; \alpha$ represents the residuals after removal of the effects of solar activity and $\alpha^{\prime}$ is the effect of geomagnetic activity on the ionosphere.

\section{Analysis and Discussion}

\subsection{Validating the Effects of Solar and Geomagnetic Activity}

Figure 2 presents the relationships between the ionospheric $f_{0} \mathrm{~F} 2$ and the solar activity index F10.7 (or the geomagnetic activity Ap index) at different LT (local time). The ionospheric foF2 observations were obtained from 27 global ionosondes (station numbers are indicated along the y axis) over a period of more than 30 years. The correlation coefficient between $f \circ F 2$ and F10.7 (Ap) is shown in Figure $2 \mathrm{a}, \mathrm{d}$. As the main driver, the effect of solar activity dominates the trend of ionospheric $f \circ \mathrm{F} 2$; the correlation between the original $f \circ \mathrm{F} 2$ values and the $\mathrm{F} 10.7$ index is very high, which are up to 0.7 in most ionospheric stations, especially during the daytime (Figure 2a). Due to the original $f o F 2$ and Ap including the data in the geomagnetic quiet period, which covers most of the data range, the correlation coefficients between $f o \mathrm{~F} 2$ and Ap are small. Therefore, the effects of geomagnetic activity are difficult to identify in the original observations (Figure 2d) and the correlation coefficient between $f_{0} \mathrm{~F} 2$ and Ap does not exceed 0.1 for most stations. After processing the original data through the traditional method (Equations (2) and (3)), as shown in Figure $2 \mathrm{~b}$, the effect of solar activity on $\Delta f o \mathrm{~F} 2$ is weakened, but the correlation coefficients between $\Delta f o \mathrm{~F} 2$ and the F10.7 index are still very high (more than 0.7 for some stations). The effects of geomagnetic activity on $\Delta f o \mathrm{~F} 2$ are weak (Figure 2e). This implies that the traditional method hardly extracts the geomagnetic activity effects. Using the SWM process (Equations (4) and (5)), the correlation coefficient between $\Delta f o F^{\prime}$ and F10.7 (Ap) indices is presented in Figure 2e,f. Compared with traditional method, this suggests that the SWM can reject the effect of solar activity (the correlation coefficient is less than 0.3 ) and better extract the effect of geomagnetic activity from the ionosphere. The SWM may be suitable in studying the geomagnetic activity on the ionosphere. In fact, solar activity not only affects the ionosphere's behavior, it also significantly modifies the variability in geomagnetic activity, so explorations of the effects of geomagnetic activity on the ionosphere should not completely neglect the variation of solar activity in different solar cycles. 


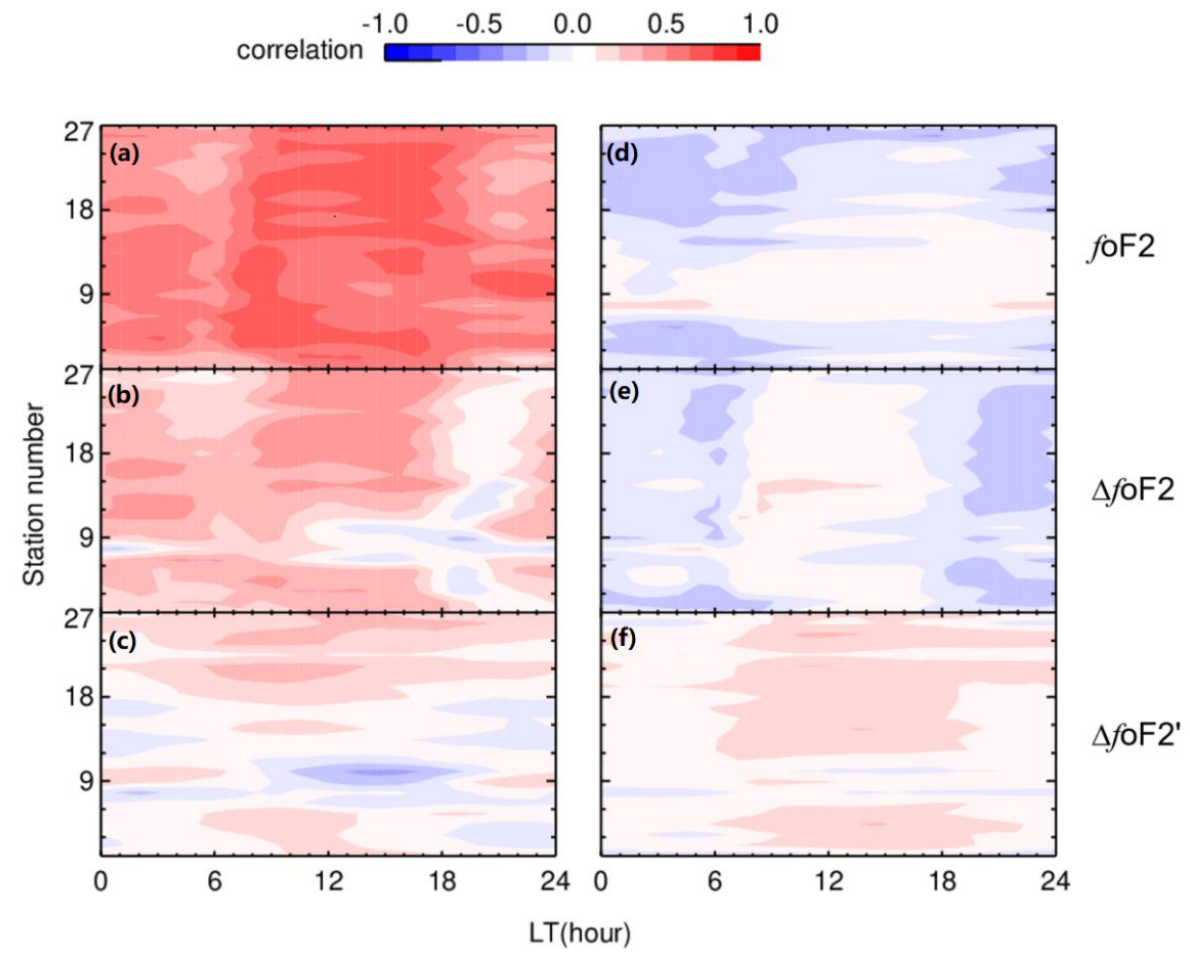

Figure 2. (left panels) Correlation coefficient (CC) between solar activity index F10.7 and ionospheric observation $f_{\mathrm{oF}}$ (panel a) or the residual of $f_{0} \mathrm{~F} 2\left(\Delta f_{0} \mathrm{~F} 2\right.$ (panel b) and $\Delta f_{0} \mathrm{~F} 2^{\prime}$ (panel c)) at different local times. (right panels) $\mathrm{CC}$ between geomagnetic index $A p$ and $f_{\mathrm{oF}} 2$ (panel d) or residual $f_{o} \mathrm{~F} 2(\Delta f o \mathrm{~F} 2$ (panel e) and $\Delta f o{ }^{\prime} 2^{\prime}($ panel $\mathbf{f})$ ).

As presented above, since SWM may be a useful method for analyzing the effects of geomagnetic activity, the SWM will be further used to study the contribution of geomagnetic activity on the ionosphere at different phases of the solar cycle. In this study, solar maxima and minima were calculated based on the 13-month mean of the International Sunspot Number [26]. The middle of the period between a given cycle's minimum and maximum was identified as an ascending year, and the equivalent middle of the period between a given cycle's maximum and the minimum was defined as a declining year. Next, the full effect of geomagnetic activity at different phases of the solar cycle was calculated using the absolute values of the ionospheric perturbations over the course of one year in each phase (maxima, minima, ascending, and declining periods). Finally, the trend of the overall effect of geomagnetic activity was calculated using a linear equation. The specific algorithm was implemented as follows:

$$
\left\{\begin{array}{l}
\operatorname{Ge}\left(T_{S}\right)=\frac{1}{T} \int_{0}^{T}|\operatorname{SWM}(g(t))| \mathrm{d} t \\
\operatorname{Ge}\left(T_{S}\right)=a_{S} \times \text { year }+\beta_{S}
\end{array} \quad(S=0,1,2,3)\right.
$$

where $g(t)$ represents the ionospheric observations; SWM $(g(t))$ refers to extraction of the effects of geomagnetic activity from ionospheric observations using the SWM; $T_{S}$ represents the different phases throughout the solar cycle $(S=0,1,2$, and 3 represent the solar maximum, solar minimum, the ascending phase, and the declining phase, respectively); $T$ is the time period covered by a given ionosonde station for one year; $\mathrm{Ge}$ is the mean level of the effect of geomagnetic activity on the ionosphere during one year of $T_{S}$; and $\alpha_{s}$ represents the ionospheric trend caused by geomagnetic activity at different phases of the solar cycle. The resulting Ge levels at different phases of the solar cycle are presented in Figure 3. The positive trend means that ionospheric variation caused by geomagnetic activity is rising with time during the period. On the other hand, the negative trend means that the effect of geomagnetic activity on the ionosphere is declining with time during the period. At the solar minimum and during the 
ascending phase, the trends are characterized by very small values $\left(-0.005\right.$ to $\left.0.005 \mathrm{MHz}_{\mathrm{year}}{ }^{-1}\right)$ and the positive trends are essentially equal to the negative trends. The effects of geomagnetic activity on ionospheric variations for the 27 stations are more dynamic and intense $\left(-0.03\right.$ to $0.03 \mathrm{MHz}$ year $\left.{ }^{-1}\right)$ during solar maximum, although the positive $(50.7 \%)$ and negative $(49.3 \%)$ trends are almost the same. During the solar maximum, the effects obviously show positive trends at higher latitudes; however, the effects show negative trends at lower latitudes (form $15^{\circ} \mathrm{S}$ to $40^{\circ} \mathrm{N}$ ). During the declining phase, it seems that the effects of geomagnetic activity on the ionospheric variations are most notable. The negative trends (11.3\%) occur significantly less frequently than positive trends $(88.7 \%)$, with an overall trend of close to $0.02 \mathrm{MHz}$ year ${ }^{-1}$. The negative trends only occur at lower latitudes in the afternoon region. For other regions, the closer the time is to noon, the stronger the positive trend is expected to be, as seen in the original data (Figure 2f), where geomagnetic activity had its greatest impact on the ionospheric trend.

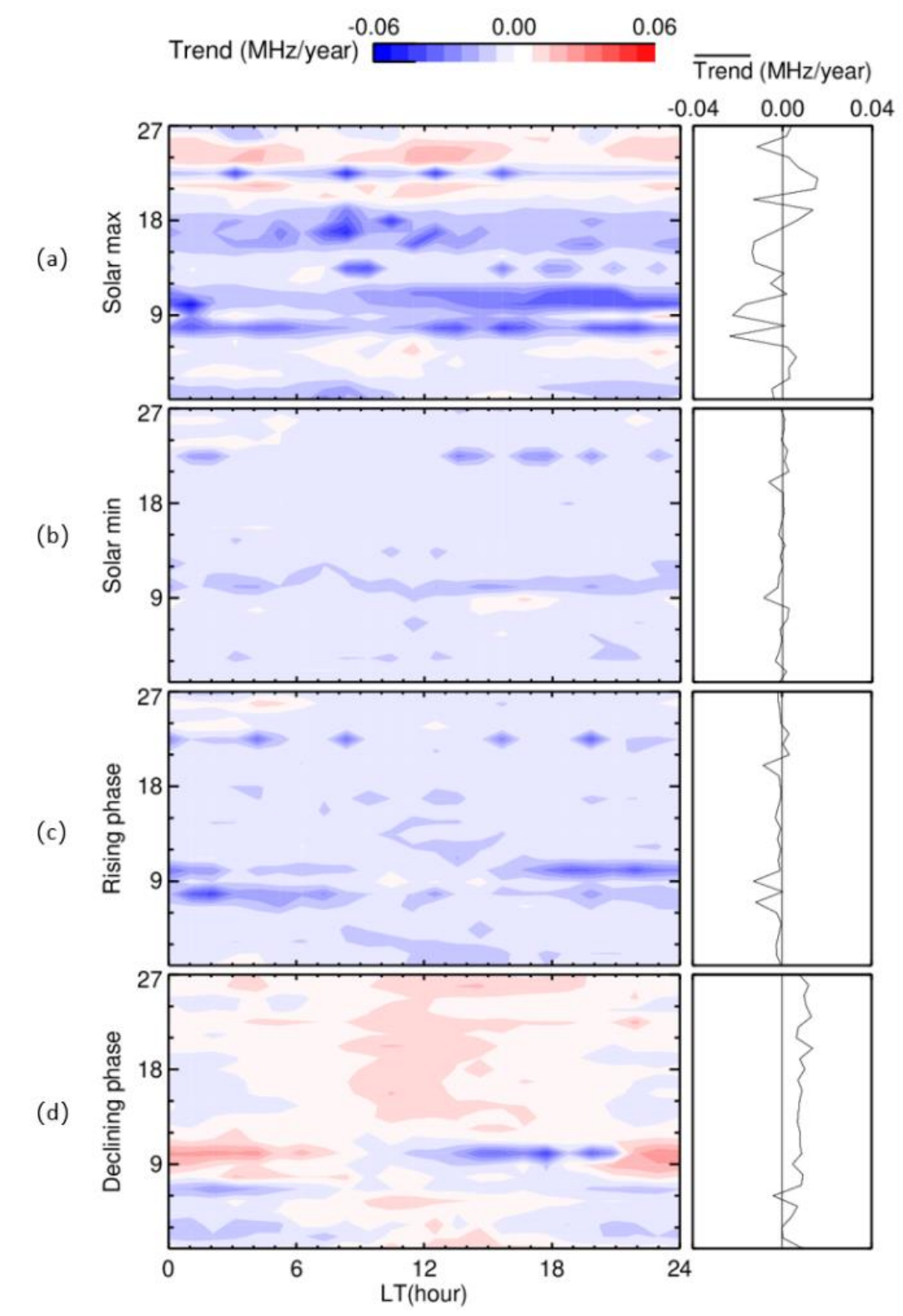

Figure 3. (left panels) $\mathrm{Ge}$ (the index reflects the effect of geomagnetic activity on ionosphere at mean level) as a function of LT (local time) at different phases of solar activity (a-d represent solar max, solar min, rising phase, and declining phase, respectively). The $x$ and $y$ axes show LT and station number, respectively. (right panels) Means of Ge over all LT values for the different stations. 
As far as we know, the response of the ionospheric F2-layer ( $f \circ \mathrm{F} 2$ ) to geomagnetic activity depends on the season and LT; thus, studying the dependence of ionospheric trends on the local time and season is necessary (as shown in Figure 4). This effect shows that the global ionosphere varies greatly with the seasonal trends at different phases of solar activity. In total, the global ionospheric trend varies hugely with different seasons, solar maxima, and declining years, but very weakly with solar minima and rising years. In general, the responses of the ionosphere to geomagnetic activity are larger around winter and smaller around summer.

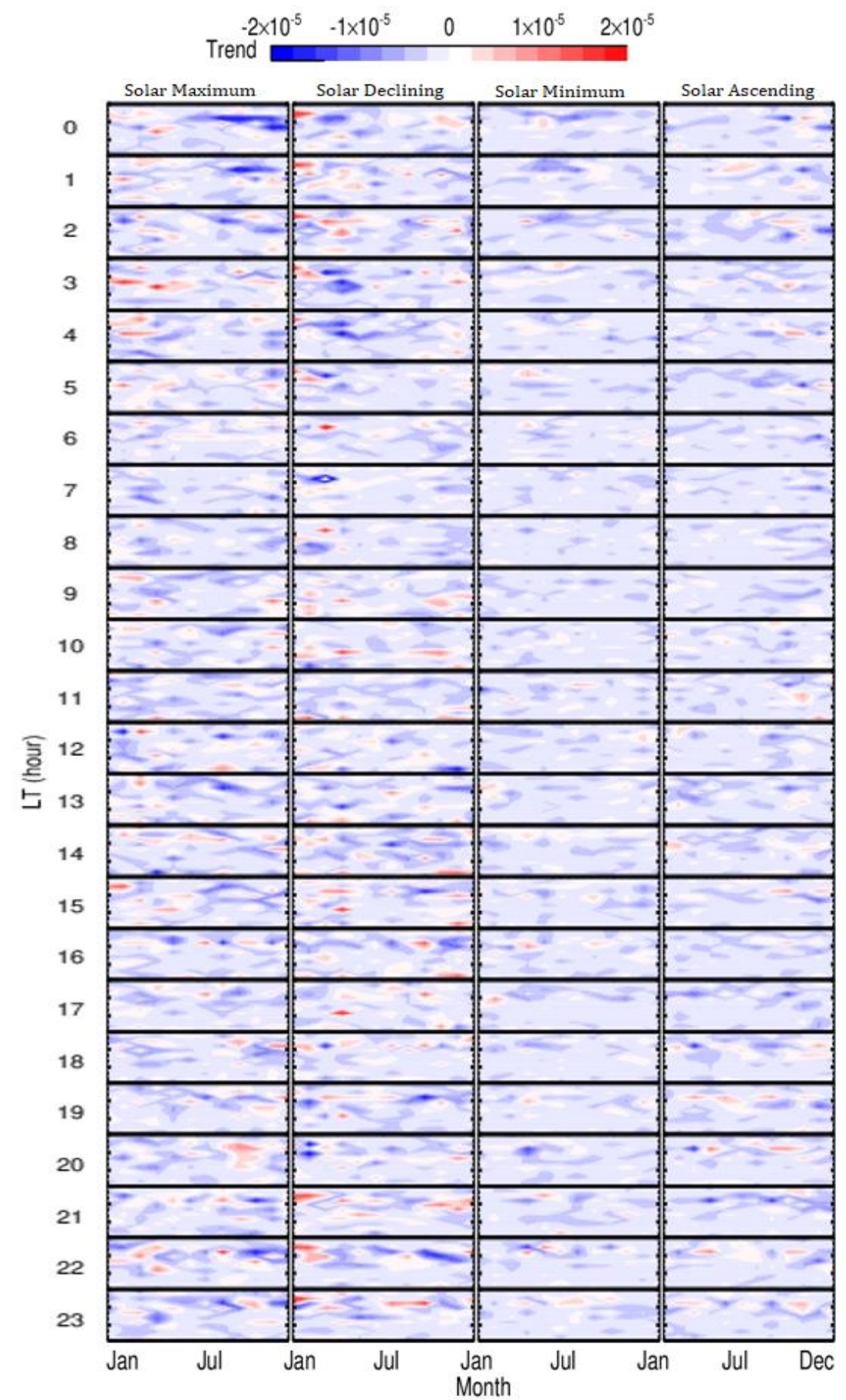

Figure 4. The effects of geomagnetic activity on global ionospheric trends depend on the local time and season. Each small graph represents the variations of the ionospheric trends caused by geomagnetic activity as a function of the month in fixed local time and solar cycle phases. 


\subsection{Discussion}

Using the SWM, we study the contribution of geomagnetic activity to the ionosphere. The result from the SWM is compared with the results from the MMM and a traditional method (as shown in Figures 1 and 2, respectively). As presented in Figure 1, the disturbances derived from the MMM have an obvious residual of diurnal variation and 27-day quasi-periodicity. However, the results from SWM can avoid these phenomena. Moreover, in the range of the global scale, as shown in Figure 2, the correlation coefficient between the $\Delta f o \mathrm{~F}^{\prime}$ (the geomagnetic effect on $f o \mathrm{~F} 2$ using the SWM) and Ap is much higher than that between $\Delta f o \mathrm{~F} 2$ (the geomagnetic effect on $f o \mathrm{~F} 2$ using a traditional method) and Ap. This suggests that the SWM can more effectively weaken the effects of solar activity and better extract the effects of geomagnetic activity. Both of the above comparisons may confirm that the SWM is a suitable method for extracting the effect of geomagnetic activity on $f o F 2$. Our previous research has also proved that the SWM is an effective method to reflect the ionospheric response to strong geomagnetic activity in special or global regions [18]. The goal of this paper is to further use this method to study the contribution of geomagnetic activity to the ionosphere in different phases of solar cycle.

As is well known, variations of ionospheric responses to geomagnetic activity significantly depend on location (longitude, latitude) and season; thus, studying global ionospheric variation responses to geomagnetic activity at different locations or in different seasons is challenging. Some related research studies have been performed previously. However, it seems that different approaches and methods can produce different results [15]. Bremer [14] found that the effects of geomagnetic activity on the F2-layer are not latitudinally dependent, but obvious longitudinal dependence can be found. However, using an improved method based on regression and the running mean, Mikhailov and Marin [15] obtained contradictory results. They suggested that the variation in the F2 layer is mainly dependent on latitude. Furthermore, Yue et al. [27] used an artificial neural net (ANN) method to explore the trends of $f_{0} \mathrm{~F} 2$ in the Asia-Pacific sector. Their resulted showed no pronounced diurnal or latitudinal variations. However, for most stations there was a pronounced seasonal effect.

Wang et al. [18] firstly introduced the SWM, which is a mathematical method. The disturbances detected by the SWM have a Gaussian-type probability density function (PDF), further simplifying the statistical analysis and suggesting that the identified absolute disturbances can be compared regardless of the time and scales. In this study, the analysis based on this filter method covers regions ranging from high to low latitudes in both hemispheres to assess the perturbation of the ionosphere in different phases of the solar cycle. Based on the SWM process, diurnal variation can be found during the solar declining phase of the solar cycle; the significant effect of geomagnetic activity on the ionosphere exhibits an obvious solar cycle phase dependence (Figure 3). This result is similar to that produced by Mikhailov and Marin [15]. However, no significant latitudinal or longitudinal dependence is shown at any phase of solar cycle, similar to the results of Yue et al. [27].

The relationship between the geomagnetic and solar activity is complex, as coronal mass ejections, high-speed streamers, and slow solar winds can affect temporal variations in geomagnetic activity over a solar cycle [28]. In fact, solar activity is an important driver of geomagnetic activity. As shown in Figure 5, the geomagnetic activity maximum always lags behind the solar activity maximum, which often appears during the declining phase of the solar activity cycle. However, the geomagnetic and solar activity minimum years occur close to each other in most solar cycles. During the solar maximum year, the annual average of the geomagnetic activity is often relatively low. Richardson et al. [28] found that this phenomenon is present from cycles 19 to 22 (hatching in Figure 5b). Such decreases in the geomagnetic activity are thought to be a manifestation of the so-called "Gnevyshev gap" [29-32], which suggests that strong solar activity modulates strong geomagnetic activity in a complicated fashion. The delay of geomagnetic activity with respect to the 11-year solar cycle has been known for many years [33]. However, as shown in Figure 5, the time scale of the delay is complex and dynamic; the delay time intervals appear to be different for different solar cycles. 


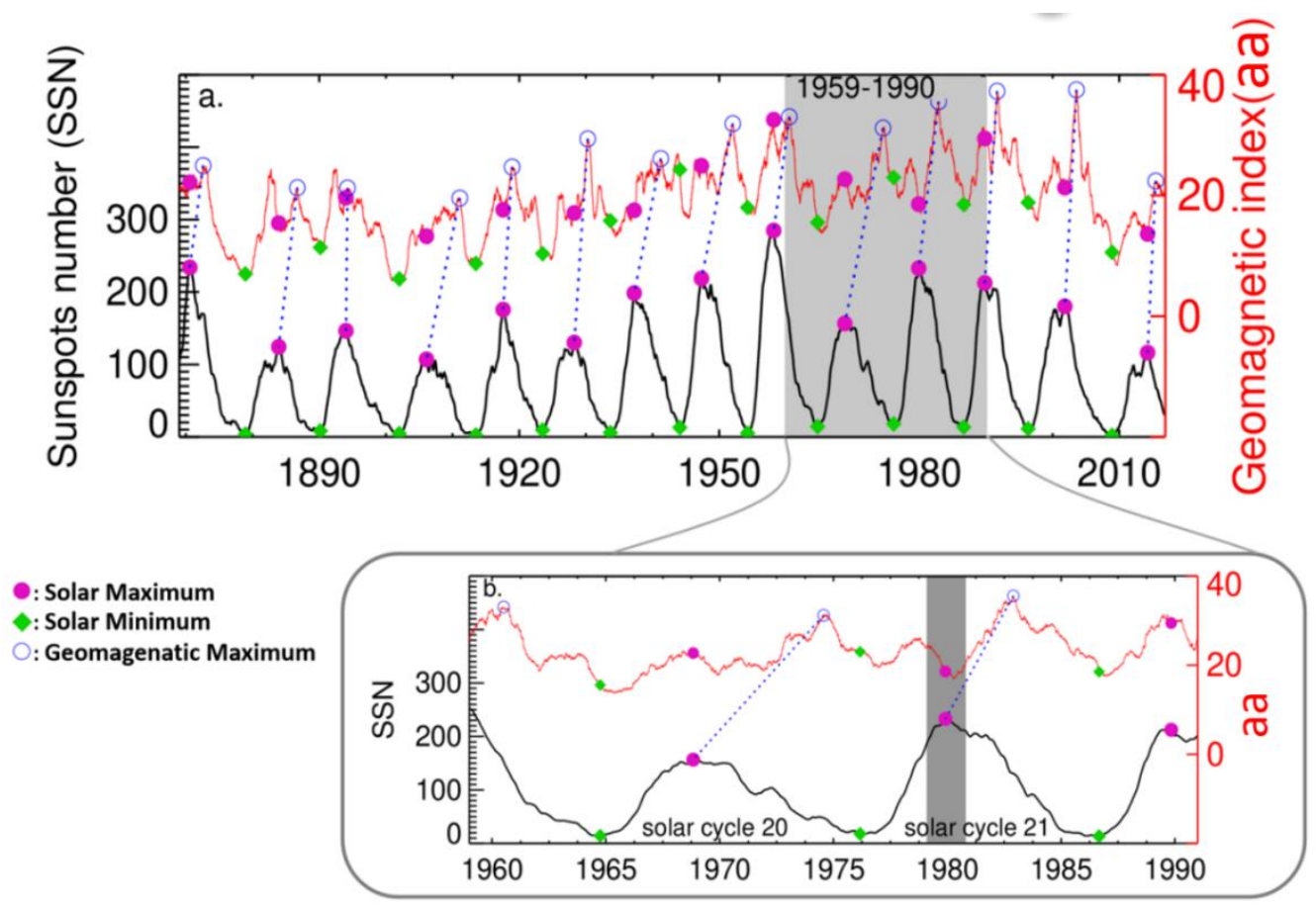

Figure 5. (a) Time variations for the sunspot number (SSN; black line) and geomagnetic index ( $a a$; red line) from 1868 to 2017. (b) SSN and aa from 1959 to 1990. Filled circles: maxima for solar activity. Filled diamonds: minima for solar activity. Open circles: maxima for geomagnetic activity.

This suggests that the trends for solar and geomagnetic activities are not synchronized and that the lag between the two can offer an opportunity to understand the contribution of geomagnetic activity to ionospheric trend variations. The geomagnetic activity affects the ionosphere differently under solar maximum and minimum periods. For solar maxima, more and stronger geomagnetic activities will mean that the effects of these activities on the ionosphere are easily identifiable. Their effects on ionospheric trends vary greatly with different stations, resulting from different regional ionosphere with different responses to geomagnetic storms [1,2]. However, during solar activity minimum years, the lower level of geomagnetic activity will contribute weakly to the long-term ionospheric trend, which will consequently have a very small value. During ascending years, the geomagnetic activity is also very weak, so its effect is the same as minimum years (Figure 3c). During a declining year, solar activity will drop to a mean level, which will have a relatively weak effect on the ionosphere, but geomagnetic activity will reach its maximum level. Thus, geomagnetic activity will play a more important role than other solar cycle phases in the ionosphere. As shown in Figure 3d, most regional variations are positive, and during daytime the trend is more significant. This is similar to the trend seen in the original data (Figure 2a). This suggests that the contribution of geomagnetic activity to the ionosphere is significant and is comparable to that of solar activity when geomagnetic activity is strong.

\section{Conclusions}

As is well known to all, the response of the ionospheric F2-layer to geomagnetic activity is very complicated. For example, with some increased geomagnetic activities, an increase in the molecular nitrogen concentration and a simultaneous decrease in the atomic oxygen concentration will result in ionospheric disturbance of the negative phase. On the other hand, the ionospheric disturbance of the positive phase includes uplifting due to vertical drifts (caused by enhanced equatorward neutral winds or increases of electric fields from the magnetosphere), plasma fluxes from the plasmasphere, and neutral composition changes. These two opposite physical processes compete with each other at large scales, which could result in diverse consequences for the ionosphere. For the above reasons, 
in order to statically demonstrate the contribution of geomagnetic activity to the ionospheric $f \circ \mathrm{F} 2$, special algorithms or methods are required. In this paper, the SWM is adapted to extract the effects of geomagnetic activity on the ionosphere. Compared to traditional methods, the SWM can more effectively weaken the contributions of solar activity and better extract the effects of geomagnetic activity.

Using the SWM, the global effect of geomagnetic activity on the ionospheric $f_{0} \mathrm{~F} 2$ in different phases (maximum, minimum, declining phase, and ascending phase) of solar activity are studied. The results suggest that this contribution to the ionosphere is significant when geomagnetic activity is at its maximum level, which usually happens in the declining phase of the solar cycle. This may be because the geomagnetic activity maximum generally lags behind the solar activity maximum, which often appears during the declining phase of the solar activity cycle. Furthermore, the time scale of the lag is complex and dynamic. On the other hand, the contribution is very weak in the solar minimum period and during the ascending phase. This suggests a complex relationship between solar activity and geomagnetic activity, with effects on the ionosphere. The results also reveal the obvious seasonal dependence of the ionospheric response; the responses of the ionosphere to geomagnetic activity are larger around winter and smaller around summer.

Author Contributions: Z.C. conceived the basic idea for the paper. H.L., J.-S.W., and Z.C. designed the experiments and analysis tools, performed the experiments, and analyzed the data. H.L., J.W., L.X., F.L., and T.Z. participated in discussion and interpretation of the data. H.L. and Z.C. wrote the paper. All authors have read and agreed to the published version of the manuscript.

Funding: This research was funded by the National Natural Science Foundation of China (grant no. 41604136, no. 41604156, no. 41774195, no. 41974183) and the Natural Science Foundation of Jiangxi Province of China (20171BAB213028).

Acknowledgments: The foF2 data used in the present paper are from Space Physics Interactive Data Resource, available online at http://www.ngdc.noaa.gov/stp/spidr.html. The F10.7 data are obtained from National Center for Environmental Information, NOAA, available online at https:/www.ngdc.noaa.gov/stp/space-weather/solar-data. The geomagnetic indices are from the World Data Center for Geomagnetism, Kyoto, are online at http://wdc.kugi. kyoto-u.ac.jp/dstae/index.html. We thank the (Space Physics Interactive Data Resource), NCEI (National Centers for Environmental Information), and the World Data Center for Geomagnetism for making their data available.

Conflicts of Interest: The authors declare no conflict of interest.

\section{References}

1. Prölss, G.W. Magnetic storm associated perturbations of the upper atmosphere. Magn. Storms 1997, 98, $227-241$. [CrossRef]

2. Fuller-Rowell, T.M.; Codrescu, M.V.; Roble, R.G.; Richmond, A.D. How Does the Thermosphere and Ionosphere React to a Geomagnetic Storm? Magn. Storms 1997, 98, 203-225. [CrossRef]

3. Zhou, M.; Li, B.; Huang, S.; Deng, X.; Ashour-Abdalla, M.; Nishimura, Y.; Yuan, Z.; Pang, Y.; Li, H. Observation of large-amplitude magnetosonic waves at dipolarization fronts. J. Geophys. Res. Space Phys. 2014, 119, 4335-4347. [CrossRef]

4. Tang, R.; Summers, D.; Deng, X. Effects of cold electron number density variation on whistler-mode wave growth. Ann. Geophys. 2014, 32, 889-898. [CrossRef]

5. Li, H.; Yuan, Z.; Yu, X.; Huang, S.; Wang, D.; Wang, Z.; Qiao, Z.; Wygant, J.R. The enhancement of cosmic radio noise absorption due to hiss-driven energetic electron precipitation during substorms. J. Geophys. Res. Space Phys. 2015, 120, 5393-5407. [CrossRef]

6. Liu, L.; Wan, W.; Ning, B.; Pirog, O.M.; Kurkin, V.I. Solar activity variations of the ionospheric peak electron density. J. Geophys. Res. 2006, 111, 304. [CrossRef]

7. Yiğit, E.; Kilcik, A.; Elias, A.G.; Dönmez, B.; Ozguc, A.; Yurchshyn, V.; Rozelot, J.P. Critical frequencies of the ionospheric F1 and F2 layers during the last four solar cycles: Sunspot group type dependencies. J. Atmos. Sol.-Terr. Phys. 2018, 171, 157-163. [CrossRef]

8. Cai, Y.; Yue, X.; Wang, W.; Zhang, S.; Liu, L.; Liu, H.; Wan, W. Long-term trend of topside ionospheric electron density derived from DMSP data during 1995-2017. J. Geophys. Res. Space Phys. 2019, 124, 10708-10727. [CrossRef] 
9. Holt, J.M.; Zhang, S.R. Long-term temperature trends in the ionosphere above Millstone Hill. Geophys. Res. Lett. 2008, 35, L05813. [CrossRef]

10. Zhong, J.; Lei, J.; Dou, X.; Yue, X. Is the long-term variation of the estimated GPS differential code biases associated with ionospheric variability? GPS Solut. 2016, 20, 313-319. [CrossRef]

11. Qian, L.; Solomon, S.C.; Roble, R.G.; Kane, T.J. Model simulations of global change in the ionosphere. Geophys. Res. Lett. 2008, 35, L07811. [CrossRef]

12. Elias, A.G.; de Adler, N.O. Earth magnetic field and geomagnetic activity effects on long term trends in the F2 layer at mid-high latitudes. J. Atmos. Sol. Terr. Phys. 2006, 68, 1871-1878. [CrossRef]

13. Mikhailov, A.V.; Marin, D. An interpretation of the foF2 and hmF2 long-term trends in the framework of the geomagnetic control conception. Ann. Geophys. 2001, 19, 733-748. [CrossRef]

14. Bremer, J. Trends in the ionospheric E and F regions over Europe. Ann. Geophys. 1998, 16, 986-996. [CrossRef]

15. Mikhailov, A.V.; Marin, D. Geomagnetic control of the $f_{0} F_{2}$ long-term trends. Ann. Geophys. 2000, 18, 653-665. [CrossRef]

16. Zhao, X.R.; Sheng, Z.; Li, J.W.; Yu, H.; Wei, K.J. Determination of the "wave turbopause" using a numerical differentiation method. J. Geophys. Res. Atmos. 2019, 124, 10592-10607. [CrossRef]

17. He, Y.; Sheng, Z.; He, M. Spectral Analysis of Gravity Waves from Near Space High-Resolution Balloon Data in Northwest China. Atmosphere 2020, 11, 133. [CrossRef]

18. Wang, J.S.; Chen, Z.; Huang, C.M. A method to identify aperiodic disturbances in the ionosphere. Ann. Geophys. 2014, 32, 563-569. [CrossRef]

19. Chen, Z.; Wang, J.S.; Deng, X.; Deng, Y.; Huang, C.M.; Li, H.M.; Wu, Z.X. Study on the relationship between the residual 27 day quasiperiodicity and ionospheric Q disturbances. J. Geophys. Res. Space Phys. 2017, 122, 2542-2550. [CrossRef]

20. Bruce, J.W.; Giblin, P.J. Curves and Singularities: A geometrical Introduction to Singularity Theory; Cambridge University Press: Cambridge, UK, 1992.

21. Chen, Z.; Wang, J.S.; Huang, C.M.; Huang, L.F. A new pair of indices to describe the relationship between ionospheric disturbances and geomagnetic activity. J. Geophys. Res. Space Phys. 2014, 119, 10156-10163. [CrossRef]

22. Chen, Z.; Wang, J.S.; Deng, Y.; Huang, C.M. Extraction of the geomagnetic activity effect from TEC data: A comparison between the spectral whitening method and 28 day running median. J. Geophys. Res. Space Phys. 2017, 122, 3632-3639. [CrossRef]

23. Upadhyay, H.O.; Mahajan, K.K. Atmospheric greenhouse effect and ionospheric trends. Geophys. Res. Lett. 1998, 25, 3375-3378. [CrossRef]

24. Zhang, S.R.; Holt, J.M. Long-term ionospheric cooling: Dependency on local time, season, solar activity, and geomagnetic activity. J. Geophys. Res. 2013, 118, 3719-3730. [CrossRef]

25. Elias, A.G.; de Haro Barbas, B.F.; Shibasaki, K.; Souza, J.R. Effect of solar cycle 23 in foF2 trend estimation. Earth Planets Space 2014, 66. [CrossRef]

26. Hathaway, D.H. The Solar Cycle. Living Rev. Sol. Phys. 2015, 12, 4. [CrossRef]

27. Yue, X.; Wan, W.; Liu, L.; Ning, B.; Zhao, B. Applying artificial neural network to derive long-term foF2 trends in the Asia/Pacific sector from ionosonde observations. J. Geophys. Res. 2006, 111, 303. [CrossRef]

28. Richardson, I.G.; Cliver, E.W.; Cane, H.V. Sources of geomagnetic activity over the solar cycle: Relative importance of coronal mass ejections, high-speed streams, and slow solar wind. J. Geophys. Res. 2000, 105, 203-213. [CrossRef]

29. Gnevyshev, M.N. On the 11-years cycle of solar activity. Sol. Phys. 1967, 1, 107-120. [CrossRef]

30. Gnevyshev, M.N. Essential features of the 11-year solar cycle. Sol. Phys. 1977, 51, 175-183. [CrossRef]

31. Feminella, F.; Storini, M. Large scale dynamical phenomena during solar activity cycles. Astron. Astrophys. 1997, 322, 311-319.

32. Richardson, I.G. Geomagnetic activity during the rising phase of solar cycle 24. J. Space Weather Space Clim. 2013, 3, A08. [CrossRef]

33. Legrand, J.P.; Simon, P.A. Ten cycles of solar and geomagnetic activity. Sol. Phys. 1981, 70, 173-195. [CrossRef]

(C) 2020 by the authors. Licensee MDPI, Basel, Switzerland. This article is an open access article distributed under the terms and conditions of the Creative Commons Attribution (CC BY) license (http://creativecommons.org/licenses/by/4.0/). 